\title{
Dynamics of medium thickness plates interacting with a periodic Winkler's foundation: non-asymptotic tolerance modelling
}

\author{
Jarosław Jędrysiak • Agnieszka Paś
}

Received: 18 August 2012/ Accepted: 24 March 2014/Published online: 10 April 2014

(C) The Author(s) 2014. This article is published with open access at Springerlink.com

\begin{abstract}
Medium thickness plates resting on a periodic Winkler's foundation are investigated. New averaged non-asymptotic models for those plates are proposed. These models are based on the tolerance averaging technique. The main feature of these models is that they describe the effect of period lengths on the overall behaviour of the plate. It is also shown that from governing equations of these models, equations of simplified averaged models (called asymptotic models) can be obtained. An additional interesting feature of the proposed models is that the equations describe also the effect of normal stress in the thickness direction.
\end{abstract}

Keywords Medium thickness plate $\cdot$ Periodic Winkler's foundation - Effect of period lengths

\section{Introduction}

Plates interacting with a subsoil are often applied as elements of constructions in the civil engineering, e.g. as elements of building foundations or reinforcements

J. Jędrysiak ( $\square)$

Department of Structural Mechanics, Łódź University of Technology, al. Politechniki 6, 90-924 Lodz, Poland e-mail: jarek@p.lodz.pl

A. Paś

Lodz, Poland

e-mail: agnieszka_pas@yahoo.ie of roads foundations. In many cases as a certain approximation the subsoil is modelled as a Winkler's foundation.

In this paper a special case of the aforementioned plates is considered, i.e. a medium thickness plate (homogeneous and anisotropic) resting on a periodic Winkler's foundation, cf. Fig. 1. Plates of this kind are used as constructions under roads, e.g. as concrete plates resting on a weak subsoil, which is reinforced by a system of periodically distributed vertical pillars, made of sand or gravel.

The above systems, i.e. plates on a periodic foundation, consist of many small identical elements, called periodicity cells. Properties of these structures are described by highly oscillating, periodic and often non-continuous functions. Because an analysis of engineering problems of these plates is too complicated using exact equations of the plate theory, different averaged models have been proposed. These models have usually described certain homogeneous plates with constant homogenized properties instead of real periodic plates. Between these models it can be mentioned those based on the method of asymptotic homogenization for periodic solids proposed in Bensoussan et al. [3]. Models of this kind for periodic plates were presented in a series of papers, e.g. Caillerie [5], Kohn and Vogelius [17]. Other models of these plates are based on the microlocal parameters approach, cf. Matysiak and Nagórko [18]. However, the aforementioned models usually neglect the effect of period lengths on the overall dynamic plate 
Fig. 1 A fragment of a medium thickness plate resting on a periodic Winkler's foundation

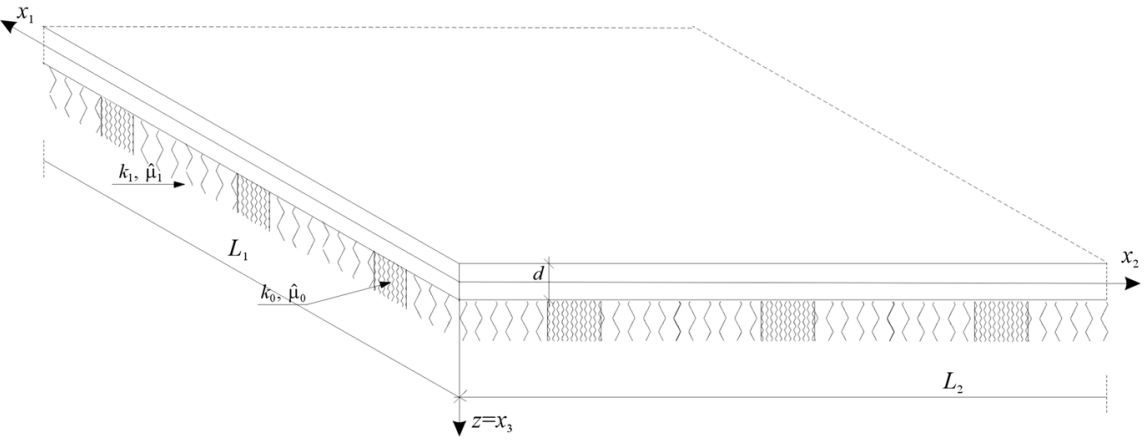

behaviour. Behaviour of functionally graded plates resting on a foundation is also analysed, e.g. Tahouneh and Naei [21], where considerations are based on the three-dimensional elasticity theory, Yajuvindra Kumar and Lal [26], where vibrations of nonhomogeneous plates with varying thickness interacting with a foundation are investigated.

In order to take into account this effect new nonasymptotic averaged models, based on the tolerance averaging technique, have been proposed. This approach was discussed for periodic composites and structures in the monograph by Woźniak and Wierzbicki [25] and in the book edited by Woźniak et al. [24]. The tolerance averaging procedure were applied to analyse non-stationary problems for different periodic structures in many papers, e.g. for periodic grounds by Dell'Isola et al. [7], for wavy plates by Michalak [19], Kirchhoff plates by Jędrysiak [11-14], for HenckyBolle plates by Baron [2], for honeycomb lattice-type plates by Cielecka and Jędrysiak [6], for thin cylindrical shells Tomczyk [22]. These papers showed that the effect of period lengths plays a crucial role in dynamics of periodic structures. Moreover, some static problems of periodic thin plates with moderately large deflections were analysed by Domagalski and Jędrysiak [8]. This modelling method was also applied to analysis some dynamical problems of functionally graded media or structures, e.g. transversally graded thin plates were considered by Kaźmierczak and Jędrysiak [16] and longitudinally graded thin plates by Michalak and Wirowski [20].

The main aim of this note is to formulate a new nonasymptotic averaged model, which describes the above effect on non-stationary problems of medium thickness plates interacting with a periodic Winkler's foundation. The peculiar feature of the proposed model is that the plane stress assumption $S_{33}=0$ is omitted, i.e. the effect of the stress $S_{33}$ is not neglected, cf. Jemielita [10].

Considerations of this contribution are based on the well-known Hencky-Bolle plate theory assumptions, cf. Bolle [4], Hencky [9], which are extended on the effect of Winkler's foundation, cf. Ambartsumyan [1], and the effect of the stress $S_{33}$, cf. Jemielita [10].

\section{Fundamental relations}

\subsection{The generalized Hencky-Bolle theory assumptions}

Let us denote by $0 x_{1} x_{2} x_{3}$ the orthogonal Cartesian coordinate system in the physical space and by $t$ the time co-ordinate. Let subscripts $\alpha, \beta, \ldots(i, j, \ldots)$ run over 1 , 2 (over $1,2,3$ ) and indices $A, B, \ldots(a, b, \ldots)$ run over $1, \ldots, N(1, \ldots, n)$. Summation convention holds for all aforementioned indices. Denote also $\mathbf{x} \equiv\left(x_{1}, x_{2}\right)$ and $z \equiv x_{3}$. Let us assume that the undeformed plate occupies the region $\Omega \equiv\{(\mathbf{x}, z):-d / 2<z<d / 2, \mathbf{x} \in$ $\Pi\}$, where $\Pi$ is the midplane with length dimensions $L_{1}, L_{2}$ along the $x_{1}$ - and $x_{2}$-axis, respectively, and $d$ is the plate thickness.

It is assumed that a plate structure, being a subject of investigations, is consisted of an anisotropic and homogeneous medium thickness plate, interacting with a periodic Winkler's foundation, which rests on a rigid undeformable base, cf. Vlasov and Leontiev [23]. A fragment of such plate is presented in Fig. 1. Hence, plate properties, i.e. a mass density $\rho$ and elastic modulae $a_{i j k l}$, are constant. Moreover, the heterogeneous foundation is periodic in planes parallel to the plate midplane, i.e. along the $x_{1}$ - and $x_{2}$-axis 
directions with periods $l_{1}$ and $l_{2}$, respectively; however, it has constant properties along the $z$-axis direction. Hence, foundation properties i.e. a mass density per an unit area $\hat{\mu}=\hat{\mu}(\mathbf{x})$ and Winkler's coefficients $k_{i}=k_{i}(\mathbf{x}), i=1, \ldots 3$, along the $x_{i}$-axis directions, can be periodic functions in $\mathbf{x}=\left(x_{1}, x_{2}\right)$. Below, it is assumed $k_{3}=k(\mathbf{x}), k_{1}=k_{2}=k_{t}(\mathbf{x})$. These foundation parameters can be defined following Vlasov and Leontiev [23]. The periodicity basic cell on $0 x_{1} x_{2}$ plane can be denoted by $\Delta \equiv\left(-l_{1} / 2, l_{1} / 2\right) \times\left(-l_{2} / 2, l_{2} / 2\right)$. The parameter $l \equiv\left(l_{1}^{2}+l_{2}^{2}\right)^{1 / 2}$ describes the cell size and satisfies the condition $d \ll l \ll L_{\min }$ ( $L_{\min }$ is a minimum characteristic length dimension of the plate in its midplane). Moreover, it is assumed that the plate cannot be torn off from the foundation.

Denote displacements, strains and stresses by $u_{i}, e_{i j}$ and $S_{i j}$, respectively; virtual displacements and virtual strains by $\bar{u}_{i}$ and $\bar{e}_{i j}$; loadings (along the $x_{i}$-axis direction) on the bottom $\Pi^{+}$and upper $\Pi^{-}$surfaces of the plate by $p_{i}^{+}$and $p_{i}^{-}$, respectively.

The problem under consideration is analysed in the framework of the generalized Hencky-Bolle plate theory. A simplified problem of this kind was presented by Jędrysiak and Paś [15], where the effect of the stress $S_{33}$ was neglected. Below, the well-known assumptions of this generalized theory are recalled.

- The kinematic constraints

$$
\begin{array}{r}
u_{\alpha}(\mathbf{x}, z, t)=z \phi_{\alpha}(\mathbf{x}, t), \quad \alpha=1,2, \\
u_{3}(\mathbf{x}, z, t)=u(\mathbf{x}, t),
\end{array}
$$

where $u(\mathbf{x}, t)$ is the deflection of the midplane, $\phi_{\alpha}(\mathbf{x}, t)$ are independent rotations; for virtual displacements we have:

$\bar{u}_{\alpha}(\mathbf{x}, z)=z \bar{\phi}_{\alpha}(\mathbf{x}), \quad \bar{u}_{3}(\mathbf{x}, z)=\bar{u}(\mathbf{x})$.

- The strain-displacement relations

$e_{i j}=u_{(i, j)}$

- The stress-strain relations (constitutive equations) (under the assumption that the plane of elastic symmetry is parallel to the plane $z=0$ )

$$
\begin{aligned}
& S_{\alpha \beta}=c_{\alpha \beta \gamma \delta} e_{\gamma \delta}+\hat{c}_{\alpha \beta 33} S_{33}, \\
& S_{\alpha 3}=c_{\alpha 3 \gamma 3} 2 e_{\gamma 3}, \\
& S_{33}=a_{\alpha \beta 33} e_{\alpha \beta},
\end{aligned}
$$

where:

$$
\begin{aligned}
c_{\alpha \beta \gamma \delta} & =a_{\alpha \beta \gamma \delta}-a_{\alpha \beta 33} a_{33 \gamma \delta} / a_{3333}, \\
c_{\alpha 3 \gamma 3} & =a_{\alpha 3 \gamma 3}-a_{\alpha 333} a_{33 \gamma 3} / a_{3333}, \\
\hat{c}_{\alpha \beta 33} & =a_{\alpha \beta 33} / a_{3333} .
\end{aligned}
$$

- The relations for "extra" stresses

$$
\begin{aligned}
& \bar{S}_{\alpha 3}=\bar{S}_{3 \alpha}=p_{\alpha}^{+}+\left(\int_{z}^{d / 2} S_{\alpha \beta} d z\right)_{, \alpha}-\ddot{\phi}_{\alpha} \int_{z}^{d / 2} z \rho d z, \\
& \bar{S}_{33}=p_{3}^{+}+\left(\int_{z}^{d / 2} \bar{S}_{\alpha 3} d z\right)_{, \alpha}-\ddot{u} \int_{z}^{d / 2} \rho d z,
\end{aligned}
$$

where $\bar{S}_{\alpha 3}, \bar{S}_{3 \alpha}, \bar{S}_{33}$ are "extra" stresses, obtained from equilibrium equations with boundary conditions on the bottom $\Pi^{+}$and upper $\Pi^{-}$surfaces of the plate, cf. Jemielita [10].

- The virtual work principle

$$
\begin{aligned}
& \int_{\Pi} \int_{-d / 2}^{d / 2} S_{i j}(\mathbf{x}, z, t) \bar{e}_{i j}(\mathbf{x}, z) d z d a \\
& +\int_{\Pi} \int_{-d / 2}^{d / 2} \rho(\mathbf{x}, z) \ddot{u}_{j}(\mathbf{x}, z, t) \bar{u}_{i}(\mathbf{x}, z) \delta_{i j} d z d a \\
& =\int_{\Pi} p_{i}^{-}(\mathbf{x}, t) \bar{u}_{i}(\mathbf{x},-d / 2) d a+\int_{\Pi} p_{i}^{+}(\mathbf{x}, t) \bar{u}_{i}(\mathbf{x}, d / 2) d a,
\end{aligned}
$$

which is satisfied for arbitrary virtual displacements described by (2), under the assumption that these displacements neglect on the plate boundary; where $d a=d x_{1} d x_{2}$ and $\bar{\phi}_{\alpha}, \bar{u}$ are sufficiently regular, independent functions.

Loadings on the bottom and upper surfaces of the plate are assumed as:

$$
\begin{aligned}
p_{i}^{+}(\mathbf{x}, t)= & q_{i}^{+}(\mathbf{x}, t)-k_{i}(\mathbf{x}) u_{i}(\mathbf{x}, d / 2, t) \\
& -\hat{\mu}(\mathbf{x}) \ddot{u}_{i}(\mathbf{x}, d / 2, t), \\
p_{i}^{-}(\mathbf{x}, t)= & q_{i}^{-}(\mathbf{x}, t),
\end{aligned}
$$

where $q_{i}^{+}(\mathbf{x}, t)$ are the parts of the loadings which are independent of the foundation; $k_{i}(\mathbf{x})$ are Winkler's coefficients $\left(k_{\alpha}(\mathbf{x})=k_{t}(\mathbf{x}), k_{3}(\mathbf{x})=k(\mathbf{x})\right)$ and $\hat{\mu}(\mathbf{x})$ is 
the mass density of the foundation per an unit area, which are determined as following Vlasov and Leontiev [23]. It can be observed that the effect of the foundation is taken into account in equation (9) by loadings on the bottom surface of the plate $p_{i}^{+}$, cf. (10) 1 .

\subsection{Equations with terms describing the effect} of the stress $\mathrm{S}_{33}$

Using the above assumptions (1)-(10) of the generalized Hencky-Bolle plate theory, after some manipulations equations of medium thickness plates resting on Winkler's foundations can be written in the form:

- Equilibrium equations

$$
\begin{aligned}
M_{\alpha \beta, \beta}-Q_{\alpha}+\frac{1}{2} d\left(p_{\alpha}^{+}-p_{\alpha}^{-}\right)-\vartheta \ddot{\phi}_{\alpha} & =0, \\
Q_{\alpha, \alpha}+p_{3}-\mu \ddot{u} & =0,
\end{aligned}
$$

- Constitutive equations

$$
\begin{aligned}
M_{\alpha \beta} & =B_{\alpha \beta \gamma \delta} \phi_{(\gamma, \delta)}+s_{\alpha \beta}+\hat{s}_{\alpha \beta}, \\
Q_{\alpha} & =D_{\alpha \beta}\left(u_{, \beta}+\phi_{\beta}\right)+\frac{1}{2} k_{\alpha \beta}^{+} d p_{\beta}^{+}+\frac{1}{2} k_{\alpha \beta}^{-} d p_{\beta}^{-},
\end{aligned}
$$

where:

- $\quad \vartheta$ and $\mu$ are the rotational inertia of the plate and the mass density per an unit area, respectively, which for the homogeneous plate with a constant thickness $d$ are given by:

$\vartheta=\frac{1}{12} \rho d^{3}, \quad \mu=\rho d$

- $\quad D_{\alpha \beta}$ and $B_{\alpha \beta \gamma \delta}$ are the tensor of shear stiffnesses and the tensor of bending stiffnesses, respectively, for the homogeneous anisotropic plate with a constant thickness $d$ given by:

$D_{\alpha \beta}=k_{\alpha \beta} d c_{\alpha 3 \beta 3}, \quad B_{\alpha \beta \gamma \delta}=\frac{1}{12} d^{3} c_{\alpha \beta \gamma \delta} ;$

- $s_{\alpha \beta}, \hat{s}_{\alpha \beta}$ are terms taking into account the effect of the stress $S_{33}$, which for the homogeneous anisotropic plate with a constant thickness $d$ are described by:
These terms are obtained from formulas (7)-(8) for "extra" stresses. The outline of this procedure can be described in the following form, $\mathrm{cf}$. Jemielita [10]. Stresses $S_{\alpha 3}$ are calculated from (7) and then stress $S_{33}$ from (8). It is caused that stresses $S_{\alpha 3}$, obtained from suitable constitutive equations, do not satisfy boundary conditions on the bottom $\Pi^{+}$and upper $\Pi^{-}$surfaces of the plate, and then a form of stress $S_{33}$ along the plate thickness is not correct.

- $k_{\alpha \beta}, k_{\alpha \beta}^{+}, k_{\alpha \beta}^{-}$are shear coefficients, for the homogeneous anisotropic plate equal:

$$
\begin{aligned}
& k_{\alpha \beta}=\frac{5}{6}, k_{11}^{+}=k_{22}^{+}=k_{11}^{-}=k_{22}^{-}=\frac{1}{6}, \\
& k_{12}^{+}=k_{12}^{-}=k_{21}^{+}=k_{21}^{-}=0 .
\end{aligned}
$$

Substituting Eq. (12) into (11) and using formulas (13)-(16) the governing equations of the homogeneous anisotropic medium thickness plates with the constant thickness resting on a periodic Winkler's foundation can be written in the form of equations of motion:

$$
\begin{aligned}
& B_{\alpha \beta \gamma \delta}\left(\phi_{\gamma, \delta}\right)_{, \beta}-D_{\alpha \beta}\left(u_{, \beta}+\phi_{\beta}\right)-\vartheta \ddot{\phi}_{\alpha}-\frac{1}{4} d^{2}\left(k_{t} \phi_{\alpha}+\hat{\mu} \ddot{\phi}_{\alpha}\right) \\
& -\frac{1}{60} d^{2} \mu \hat{c}_{\alpha \beta 33} \ddot{u}_{, \beta}-\frac{1}{10} d^{2} \hat{c}_{\alpha \beta 33}(k u+\hat{\mu} \ddot{u})_{, \beta} \\
& -\frac{1}{240} d^{4} \hat{c}_{\alpha \beta 33}\left(k_{t} \phi_{\gamma}+\hat{\mu} \ddot{\phi}_{\gamma}\right)_{, \gamma \beta}+\underline{\frac{1}{24} d^{2}\left(k \phi_{\beta}+\hat{\mu} \ddot{\phi}_{\beta}\right) \delta_{\alpha \beta}} \\
& =-\frac{1}{10} d^{2}\left[q_{3}^{+}+q_{3}^{-}+\frac{1}{12} d\left(q_{\gamma}^{+}+q_{\gamma}^{-}\right)_{, \gamma}\right]_{, \beta} \hat{c}_{\alpha \beta 33}
\end{aligned}
$$

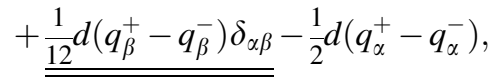

$$
\begin{aligned}
& D_{\alpha \beta}\left(u_{, \beta}+\phi_{\beta}\right)_{, \alpha}-\mu \ddot{u}-k u-\hat{\mu} \ddot{u} \\
& -\underline{\underline{\frac{1}{24} d^{2}\left(k_{t} \phi_{\beta}+\hat{\mu} \ddot{\phi}_{\beta}\right)_{, \alpha} \delta_{\alpha \beta}}}=-\underline{\underline{\frac{1}{12} d\left(q_{\beta}^{+}-q_{\beta}^{-}\right)_{, \alpha} \delta_{\alpha \beta}}} \\
& -\left(q_{3}^{+}+q_{3}^{-}\right) \text {. }
\end{aligned}
$$

The characteristic feature of equations (17) is that for periodic structures under consideration these equations have highly oscillating, periodic, functional and, in general, non-continuous coefficients, which describe the effect of the periodic foundation: $k, k_{t}, \hat{\mu}$. Moreover, underlined terms describe the effect of the stress $S_{33}$

$\begin{array}{ll}s_{\alpha \beta}=\xi \hat{c}_{\alpha \beta 33}, & \xi=\frac{1}{10} d\left[q_{3}^{+}-(k u+\hat{\mu} \ddot{u})+q_{3}^{-}\right]+\frac{1}{120} d^{2}\left[q_{\gamma}^{+}-\frac{1}{2} d\left(k_{t} \varphi_{\gamma}+\hat{\mu} \ddot{\phi}_{\gamma}\right)+q_{\gamma}^{-}\right]_{, \gamma} . \\ \hat{s}_{\alpha \beta}=\hat{\xi} d \hat{c}_{\alpha \beta 33}, & \hat{\xi}=\left(\frac{1}{40} d \mu-\frac{1}{2} d^{-1} \vartheta\right) \ddot{u}=-\frac{1}{60} \rho d^{2} \ddot{u}\end{array}$ 
and double-underlined terms - the corrected effect of the shear. Because the direct application of equations (17) to special problems is difficult, the equations are approximated by equations with constant coefficients. In order to take into account the effect of the period lengths on the overall dynamic behaviour of medium thickness plates on a periodic foundation the tolerance averaging technique will be applied.

\section{Tolerance averaging technique}

\subsection{Introductory concepts}

In order to obtain the governing equations with constant coefficients, describing the effect of period lengths, the tolerance averaging method (cf. Woźniak and Wierzbicki [25], Woźniak et al. [24]) will be applied to the equations of motion for homogeneous medium thickness plates resting on a periodic Winkler's foundation. Using the modelling procedure some concepts introduced in these books will be employed, i.e. an averaging operator, a tolerance system, a slowly-varying function, a periodic-like function, an oscillating function. Some of them are recalled below (cf. Woźniak and Wierzbicki [25] ).

Let us denote a periodicity cell at $\mathbf{x} \in \Pi_{\Delta}$, $\Pi_{\Delta}=\{\mathbf{x}: \mathbf{x} \in \Pi, \Delta(\mathbf{x}) \subset \Pi\}$, by $\Delta(\mathbf{x}) \equiv \mathbf{x}+\Delta$. The averaging operator for considered periodic systems plates-foundation is defined as

$$
\begin{aligned}
& \langle\varphi\rangle=\langle\varphi\rangle(\mathbf{x}) \equiv \\
& \left(l_{1} l_{2}\right)^{-1} \int_{\Delta(\mathbf{x})} \varphi(\mathbf{y}) d \mathbf{y}, \mathbf{x} \in \Pi_{\Delta}, \mathbf{y} \in \Delta(\mathbf{x})
\end{aligned}
$$

where $\varphi$ is an arbitrary integrable function on the midplane $\Pi$. For periodic function $\varphi$ its averaged value by (18) is constant.

Let function $F(\cdot) \in \mathrm{F}$, where $\mathrm{F}$ is a set of unknown functions in the problem under consideration, be a continuous, bounded function defined on the midplane $\Pi$. Function $F(\cdot)$ (with its derivatives) is called a slowly-varying function, $F \in S V_{\Delta}$, if $\forall x_{1}, x_{2} \in \operatorname{dom} F(\cdot) \quad|| x_{1}-x_{2} \| \leq l \Rightarrow\left|F\left(x_{1}\right)-F\left(x_{2}\right)\right| \leq \varepsilon_{F}$, where $l$ is the diameter of the cell (the parameter of a periodic structure), $\varepsilon_{F}$ is the tolerance parameter.

Let $f$ be a bounded function defined on $\Pi$ and $\phi$ be a periodic function. Function $f$ is called a periodic-like function, if for every $\mathbf{x} \in \Pi_{\Delta}$ a periodic function $f_{\mathbf{x}}$ exists such that the condition $\langle\phi f\rangle(\mathbf{x}) \cong\left\langle\phi f_{\mathbf{x}}\right\rangle$ (x) holds. Hence, function $f_{\mathbf{x}}$ is referred to as a periodic approximation of $f$ at $\mathbf{x}$. If the above condition is satisfied for all derivatives of function $f$, it can be denoted $f \in P L_{\Delta}$.

A periodic-like function $f$ will be called an oscillating function, $f \in P L_{\Delta}^{\mu}$, with the weight $\mu$, if for every $\mathbf{x} \in \Pi_{\Delta}$ it satisfies the condition $\langle\mu f\rangle(\mathbf{x}) \cong 0$, where $\mu(\cdot)$ is a positive value periodic function. For constant values functions $\mu$ the condition takes the form $\mu\langle f\rangle(\mathbf{x})=\langle f\rangle(\mathbf{x}) \cong 0$ for every $\mathbf{x} \in \Pi_{\Delta}$, and set of those functions is denoted by $f \in P L_{\Delta}^{1}$.

In the aforementioned books there are shown that these definitions are related to a periodicity cell $\Delta$ and a certain tolerance system $T$.

The above concepts together with lemmas and assertions, formulated and proved in the book by Woźniak and Wierzbicki [25] are used in the modelling procedure.

\subsection{Modelling assumptions}

In the tolerance averaging technique there are formulated two fundamental assumptions.

The Micro-Macro Decomposition (MMD) states that the generalized displacements-the deflection $u$ and the rotations $\phi_{\alpha}$-of the homogeneous medium thickness plate on a periodic foundation, can be decomposed in the following form:

$$
\begin{array}{ll}
u(\mathbf{x}, t)=w(\mathbf{x}, t)+g^{A}(\mathbf{x}) V^{A}(\mathbf{x}, t), & A=1, \ldots, N, \\
\phi_{\alpha}(\mathbf{x}, t)=\varphi_{\alpha}(\mathbf{x}, t)+h^{a}(\mathbf{x}) \Phi_{\alpha}^{a}(\mathbf{x}, t), & a=1, \ldots, n,
\end{array}
$$

where $\varphi_{\alpha}$ and $w$ are averaged parts of the rotations and the deflection, called the macrorotations and the macrodeflection, respectively; functions $g^{A}, h^{a}$ are known and can be obtained from periodic problems for the periodicity cell. They are called fluctuation shape functions. Functions $g^{A}$ or $h^{a}$ stand the system of $N$ or $n$ linear-independent periodic functions, such that $\left\langle g^{A}\right\rangle=0$ and $g^{A}(\cdot), l g_{, \alpha}^{A}(\cdot) \in O(l)$ or $\left\langle h^{a}\right\rangle=0$ and $h^{a}(\cdot), l h_{, \alpha}^{a}(\cdot) \in O(l)$. The fluctuation shape functions approximate the expected form of the oscillating part of free vibration modes of the periodic structure of the plate, cf. Jędrysiak and Paś [15]. On the other side, slowly-varying functions $\Phi_{\alpha}^{a}(\cdot, t), V^{A}(\cdot, t) \in S V_{\Delta}$ are new kinematic unknowns, called the fluctuation 
amplitudes for the rotations and for the deflection, respectively.

The Tolerance Averaging Approximation (TAA) states that in the framework of the tolerance averaging highly-oscillating non-continuous coefficients to averaged equations of the tolerance model of medium thickness plates resting on a periodic foundation with stress $S_{33}$, which under the following denotations:

$$
\begin{array}{cccc}
K_{t} \equiv\left\langle k_{t}\right\rangle, & K_{t}^{A} \equiv l^{-1}\left\langle k_{t} g^{A}\right\rangle, & \tilde{K}_{t}^{a} \equiv l^{-1}\left\langle k_{t} h^{a}\right\rangle, & \tilde{K}_{t}^{a b} \equiv l^{-2}\left\langle k_{t} h^{a} h^{b}\right\rangle \\
K \equiv\langle k\rangle, & K^{A} \equiv l^{-1}\left\langle k g^{A}\right\rangle, & K^{A B} \equiv l^{-2}\left\langle k g^{A} g^{B}\right\rangle, & \tilde{K}^{a} \equiv l^{-1}\left\langle k h^{a}\right\rangle \\
\tilde{K}^{a b} \equiv l^{-2}\left\langle k h^{a} h^{b}\right\rangle, & & \\
K_{t \alpha}^{A} \equiv\left\langle k_{t} g_{, \alpha}^{A}\right\rangle, & \tilde{K}_{\alpha}^{A a} \equiv l^{-1}\left\langle k g^{A} h_{, \alpha}^{a}\right\rangle, & \tilde{K}_{\alpha}^{a} \equiv\left\langle k h_{, \alpha}^{a}\right\rangle, & \tilde{K}_{t \alpha}^{a A} \equiv l^{-1}\left\langle k_{t} h^{a} g_{, \alpha}^{A}\right\rangle, \\
m \equiv\langle\hat{\mu}\rangle, & m^{A} \equiv l^{-1}\left\langle\hat{\mu} g^{A}\right\rangle, & m^{A B} \equiv l^{-2}\left\langle\hat{\mu} g^{A} g^{B}\right\rangle, & \\
\tilde{m}^{a} \equiv l^{-1}\left\langle\hat{\mu} h^{a}\right\rangle, & \tilde{m}^{a b} \equiv l^{-2}\left\langle\hat{\mu} h^{a} h^{b}\right\rangle, & \\
\tilde{m}_{\alpha}^{a} \equiv\left\langle\hat{\mu} h_{, \alpha}^{a}\right\rangle, & \tilde{m}_{\alpha}^{A a} \equiv l^{-1}\left\langle\hat{\mu} g^{A} h_{, \alpha}^{a}\right\rangle, & m_{\alpha}^{A} \equiv\left\langle\hat{\mu} g_{, \alpha}^{A}\right\rangle, & \tilde{m}_{\alpha}^{a A} \equiv l^{-1}\left\langle\hat{\mu} h^{a} g_{, \alpha}^{A}\right\rangle \\
H^{a b} \equiv l^{-2}\left\langle h^{a} h^{b}\right\rangle, & G^{A B} \equiv l^{-2}\left\langle g^{A} g^{B}\right\rangle, & H_{\alpha \beta}^{a b} \equiv\left\langle h_{, \alpha}^{a} h_{, \beta}^{b}\right\rangle, & G_{\alpha \beta}^{A B} \equiv\left\langle g_{, \alpha}^{A} g_{, \beta}^{B}\right\rangle, \\
\tilde{G}_{\alpha}^{A a} \equiv l^{-1}\left\langle g^{A} h_{, \alpha}^{a}\right\rangle, & \tilde{H}_{\alpha}^{a A} \equiv l^{-1}\left\langle h^{a} g_{, \alpha}^{A}\right\rangle, & & \\
C_{\alpha \beta} \equiv \hat{c}_{\alpha \beta 33} & &
\end{array}
$$

technique terms of an order $O(\varepsilon)$ in the modelling procedure have to be neglected.

\subsection{The modelling procedure}

In the framework of the tolerance averaging technique and following the book by Woźniak and Wierzbicki [25] the modelling procedure we can divide into four steps.

In the first step we substitute relations (19) into equations (17). Then, in the second step we average the resulting equations by applying (18) and using TAA (cf. Jędrysiak [12]), deriving equations for the macrodeflection $w$ and the macrorotations $\varphi_{\alpha}$.

In the third step we multiply equations (17) by test functions $\left(h^{b}, b=1, \ldots, n\right.$, for $(17)_{1}, g^{B}, B=1, \ldots, N$, for $(17)_{2}$ ), then we substitute (19) into equations. In the last step, after averaging (18) and some manipulations we arrive at additional governing equations for the fluctuation variables $\Phi_{\alpha}^{a}$ and $V^{A}$.

These governing equations are presented in the subsequent sections.

\section{Equations of the tolerance model}

4.1 The tolerance model

The aforementioned procedure of the tolerance averaging technique leads from equations (17) with can be written in the form:

$$
\begin{aligned}
& B_{\alpha \beta \gamma \delta} \varphi_{\gamma, \delta \beta}-\frac{1}{60} d^{2} C_{\alpha \beta} \mu \ddot{w}_{, \beta}-D_{\alpha \beta}\left(w_{, \beta}+\varphi_{\beta}\right)-\vartheta \ddot{\varphi}_{\alpha} \\
& -\frac{1}{10} d^{2} C_{\alpha \beta}\left(K w_{, \beta}+m \ddot{w}_{, \beta}+l K^{A} V_{, \beta}^{A}+\operatorname{lm}^{A} \ddot{V}_{, \beta}^{A}\right) \\
& +\left(\underline{\frac{1}{24}}-\frac{1}{4}\right) d^{2}\left(K_{t} \varphi_{\alpha}+m \ddot{\varphi}_{\alpha}+l \tilde{K}_{t}^{a} \Phi_{\alpha}^{a}+l \tilde{m}^{a} \ddot{\Phi}_{\alpha}^{a}\right) \\
& =-\frac{1}{10} d^{2} C_{\alpha \beta}\left[\left(q_{3}^{+}+q_{3}^{-}\right)_{, \beta}+\frac{1}{12} d\left(q_{\gamma}^{+}+q_{\gamma}^{-}\right)_{, \gamma \beta}\right] \\
& +\left(\frac{1}{12}-\frac{1}{2}\right) d\left(q_{\alpha}^{+}-q_{\alpha}^{-}\right) \text {, } \\
& D_{\alpha \beta}\left(w_{, \alpha \beta}+\varphi_{\beta, \alpha}\right)-(\mu+m) \ddot{w}-K w-l\left(m^{A} \ddot{V}^{A}+K^{A} V^{A}\right) \\
& -\frac{1}{24} d^{2}\left(K_{t} \varphi_{\beta, \alpha}+m \ddot{\varphi}_{\beta, \alpha}+l \tilde{K}_{t}^{a} \Phi_{\beta, \alpha}^{a}+l \tilde{m}^{a} \ddot{\Phi}_{\beta, \alpha}^{a}\right) \delta_{\alpha \beta} \\
& =-\frac{1}{12} d\left(q_{\beta, \alpha}^{+}-q_{\beta, \alpha}^{-}\right) \delta_{\alpha \beta}-\left(q_{3}^{+}+q_{3}^{-}\right) \text {, }
\end{aligned}
$$

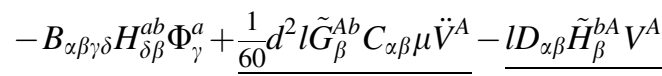

$$
\begin{aligned}
& -l^{2} H^{a b}\left(D_{\alpha \beta} \Phi_{\beta}^{a}+\vartheta \ddot{\Phi}_{\alpha}^{a}\right) \\
& +\frac{1}{10} d^{2} C_{\alpha \beta}\left(\underline{\tilde{K}_{\beta}^{b} w}+\underline{\tilde{m}_{\beta}^{b} \ddot{w}}+\underline{l \tilde{K}_{\beta}^{A b} V^{A}}+\underline{\left.l \tilde{m}_{\beta}^{A b} \ddot{V}^{A}\right)}\right. \\
& +\left(\underline{\frac{1}{24}}-\frac{1}{4}\right) d^{2} l\left(\tilde{K}_{t}^{b} \varphi_{\alpha}+\tilde{m}^{b} \ddot{\varphi}_{\alpha}+l \tilde{K}_{t}^{a b} \Phi_{\alpha}^{a}+l \tilde{m}^{a b} \ddot{\Phi}_{\alpha}^{a}\right)=0, \\
& -D_{\alpha \beta} G_{\alpha \beta}^{A B} V^{A}-l \tilde{H}_{\alpha}^{a B} D_{\alpha \beta} \Phi_{\beta}^{a}-l^{2} G^{A B} \mu \ddot{V}^{A} \\
& +\frac{1}{24} d^{2}\left(\underline{K_{t \alpha}^{B} \varphi_{\beta}}+\underline{m_{\alpha}^{B} \ddot{\varphi}_{\beta}}+\underline{l \tilde{K}_{t \alpha}^{a B} \Phi_{\beta}^{a}}+\underline{l \tilde{m}_{\alpha}^{a B} \ddot{\Phi}_{\beta}^{a}}\right) \delta_{\alpha \beta} \\
& -l\left(K^{B} w+m^{B} \ddot{w}\right)-l^{2}\left(K^{A B} V^{A}+m^{A B} \ddot{V}^{A}\right)=0,
\end{aligned}
$$

where $w, \varphi_{\alpha}, V^{A}, \Phi_{\alpha}^{a}$ are the basic unknowns, being slowly-varying functions. 
The characteristic features of the derived governing equations (21) are:

$1^{\circ}$ Constant coefficients;

$2^{\circ}$ Equations describe the effect of period lengths by terms involving parameter $l$;

$3^{\circ}$ Terms with coefficients $C_{\alpha \beta} \quad(\alpha, \beta=1,2)$ describe the effect of stress $S_{33}$;

$4^{\circ} \quad$ Terms with the underlined ratios $\frac{1}{24}$ and $\frac{1}{12}$ describe the corrected effect of the shear;

$5^{\circ}$ Terms with the coefficient $\vartheta$ describe the rotational inertia of the plate;

$6^{\circ}$ Terms with the coefficient $\hat{\mu}$ (cf. (20)) describe the inertia of the foundation.

Recapitulating, the tolerance model is defined by:

$1^{\circ}$ Equation (21) for $N+1$ and $2(n+1)$ unknowns, $w, \quad V^{A}, \quad A=1, \ldots, N$, and $\varphi_{\alpha}, \Phi_{\alpha}^{a}, \quad \alpha=1,2$, $a=1, \ldots, n$, which make it possible to analyse the effect of period lengths on the overall behaviour of the system - a medium thickness plate-a periodic Winkler's foundation;

$2^{\circ}$ Conditions determining applications of the model, i.e. equations (21) have physical sense for the unknowns $w(\cdot, t), \quad V^{A}(\cdot, t)$ and $\varphi_{\alpha}(\cdot, t), \Phi_{\alpha}^{a}(\cdot, t)$, being slowly-varying functions for every $t$;

$3^{\circ}$ The plate deflection, which is approximated by means of the formula

$u(\mathbf{x}, t)=w(\mathbf{x}, t)+g^{A}(\mathbf{x}) V^{A}(\mathbf{x}, t)$

and the plate rotations, which are given by

$\phi_{\alpha}(\mathbf{x}, t)=\varphi_{\alpha}(\mathbf{x}, t)+h^{a}(\mathbf{x}) \Phi_{\alpha}^{a}(\mathbf{x}, t)$,

where $A=1, \ldots, N, a=1, \ldots, n$.

It has to be emphasized that the above equations can be obtained for the certain mode-shape functions $g^{A}$, $A=1, \ldots, N$, and $h^{a}, a=1, \ldots, n$, which have to be previously derived for every periodic system platefoundation under consideration as solutions to certain periodic problems for the periodicity cell. In most cases our considerations can be restricted to approximate forms of these solutions and to single mode shapes for $N=1, n=1$. It makes it possible to show that it is sufficient from the computational point of view.
Neglecting in equations (21) some terms the governing equations of simplified tolerance models can be obtained.

4.2 The simplified tolerance model without the inertia of the foundation

If terms with coefficients $m, m^{A}, m^{A B}, \tilde{m}^{a}, \tilde{m}^{a b}$, $\tilde{m}_{\alpha}^{a}, \tilde{m}_{\alpha}^{A a}, m_{\alpha}^{A}, \tilde{m}_{\alpha}^{a A}$ are omitted in equations (21) we obtain:

$$
\begin{aligned}
& B_{\alpha \beta \gamma \delta} \varphi_{\gamma, \delta \beta}-\frac{1}{60} d^{2} C_{\alpha \beta} \mu \ddot{w}_{, \beta}-D_{\alpha \beta}\left(w_{, \beta}+\varphi_{\beta}\right)-\vartheta \ddot{\varphi}_{\alpha} \\
& -\frac{1}{10} d^{2} C_{\alpha \beta}\left(K w_{, \beta}+l K^{A} V_{, \beta}^{A}\right)+\left(\frac{1}{24}-\frac{1}{4}\right) d^{2}\left(K_{t} \varphi_{\alpha}+l \tilde{K}_{t}^{a} \Phi_{\alpha}^{a}\right) \\
& =-\frac{1}{10} d^{2} C_{\alpha \beta}\left[\left(q_{3}^{+}+q_{3}^{-}\right)_{, \beta}+\frac{1}{12} d\left(q_{\gamma}^{+}+q_{\gamma}^{-}\right)_{, \gamma \beta}\right] \\
& +\left(\frac{1}{12}-\frac{1}{2}\right) d\left(q_{\alpha}^{+}-q_{\alpha}^{-}\right) \text {, } \\
& D_{\alpha \beta}\left(w_{, \alpha \beta}+\varphi_{\beta, \alpha}\right)-\mu \ddot{w}-K w-l K^{A} V^{A} \\
& -\frac{1}{\underline{24}} d^{2}\left(K_{t} \varphi_{\beta, \alpha}+l \tilde{K}_{t}^{a} \Phi_{\beta, \alpha}^{a}\right) \delta_{\alpha \beta}=-\frac{1}{\underline{12}} d\left(q_{\beta, \alpha}^{+}-q_{\beta, \alpha}^{-}\right) \delta_{\alpha \beta} \\
& -\left(q_{3}^{+}+q_{3}^{-}\right),-B_{\alpha \beta \gamma \delta} H_{\delta \beta}^{a b} \Phi_{\gamma}^{a}+\frac{1}{60} d^{2} l \tilde{G}_{\beta}^{A b} C_{\alpha \beta} \mu \ddot{V}^{A}
\end{aligned}
$$

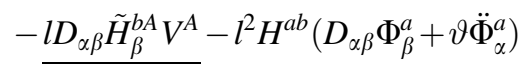

$$
\begin{aligned}
& +\frac{1}{10} d^{2} C_{\alpha \beta}\left(\tilde{K}_{\beta}^{b} w+l \tilde{K}_{\beta}^{A b} V^{A}\right) \\
& +\left(\frac{1}{24}-\frac{1}{4}\right) d^{2} l\left(\tilde{K}_{t}^{b} \varphi_{\alpha}+l \tilde{K}_{t}^{a b} \Phi_{\alpha}^{a}\right)=0, \\
& -D_{\alpha \beta} G_{\alpha \beta}^{A B} V^{A}-l D_{\alpha \beta} \tilde{H}_{\alpha}^{a B} \Phi_{\beta}^{a}-l^{2} G^{A B} \mu \ddot{V}^{A} \\
& +\frac{1}{24} d^{2}\left(\underline{K_{t \alpha}^{B} \varphi_{\beta}}+\underline{l \tilde{K}_{t \alpha}^{a B} \Phi_{\beta}^{a}}\right) \delta_{\alpha \beta}-l K^{B} w-l^{2} K^{A B} V^{A}=0,
\end{aligned}
$$

which describe the simplified tolerance model without the inertia of the foundation.

\subsection{The simplified tolerance model without the inertia of the foundation and the rotational inertia of the plate}

Neglecting in equations (21) terms with coefficients $m, m^{A}, m^{A B}, \tilde{m}^{a}, \tilde{m}^{a b}, \tilde{m}_{\alpha}^{a}, \tilde{m}_{\alpha}^{A a}, m_{\alpha}^{A}, \tilde{m}_{\alpha}^{a A}$ and also with the coefficient $\vartheta$ we arrive at: 


$$
\begin{aligned}
& B_{\alpha \beta \gamma \delta} \varphi_{\gamma, \delta \beta}-\frac{1}{60} d^{2} C_{\alpha \beta} \mu \ddot{w}_{, \beta}-D_{\alpha \beta}\left(w_{, \beta}+\varphi_{\beta}\right) \\
& -\frac{1}{10} d^{2} C_{\alpha \beta}\left(K w_{, \beta}+l K^{A} V_{, \beta}^{A}\right) \\
& +\left(\frac{1}{24}-\frac{1}{4}\right) d^{2}\left(K_{t} \varphi_{\alpha}+l \tilde{K}_{t}^{a} \Phi_{\alpha}^{a}\right)=-\frac{1}{10} d^{2} C_{\alpha \beta}\left[\left(q_{3}^{+}+q_{3}^{-}\right)_{, \beta}\right. \\
& \left.+\frac{1}{12} d\left(q_{\gamma}^{+}+q_{\gamma}^{-}\right)_{, \gamma \beta}\right]+\left(\frac{1}{12}-\frac{1}{2}\right) d\left(q_{\alpha}^{+}-q_{\alpha}^{-}\right), \\
& D_{\alpha \beta}\left(w_{, \alpha \beta}+\varphi_{\beta, \alpha}\right)-\mu \ddot{w}-K w-l K^{A} V^{A} \\
& -\frac{1}{24} d^{2}\left(K_{t} \varphi_{\beta, \alpha}+l \tilde{K}_{t}^{a} \Phi_{\beta, \alpha}^{a}\right) \delta_{\alpha \beta}=\frac{1}{\underline{12}} d\left(q_{\beta, \alpha}^{-}-q_{\beta, \alpha}^{+}\right) \delta_{\alpha \beta} \\
& -\left(q_{3}^{+}+q_{3}^{-}\right),-B_{\alpha \beta \gamma \delta} H_{\delta \beta}^{a b} \Phi_{\gamma}^{a}+\frac{1}{60} d^{2} l \tilde{G}_{\beta}^{A b} C_{\alpha \beta} \mu \ddot{V}^{A}
\end{aligned}
$$

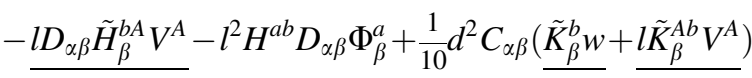

$$
\begin{aligned}
& +\left(\frac{1}{\underline{24}}-\frac{1}{4}\right) d^{2} l\left(\tilde{K}_{t}^{b} \varphi_{\alpha}+l \tilde{K}_{t}^{a b} \Phi_{\alpha}^{a}\right)=0,-D_{\alpha \beta} G_{\alpha \beta}^{A B} V^{A}
\end{aligned}
$$

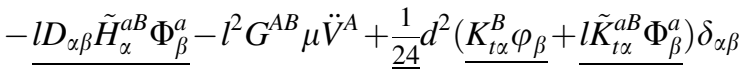

$$
\begin{aligned}
& -l K^{B} w-l^{2} K^{A B} V^{A}=0,
\end{aligned}
$$

which describe the simplified tolerance model without the inertia of the foundation and the rotational inertia of the plate.

The characteristic feature of equations (23) is that they involve time derivatives only for unknowns: $w$ and $V^{A}, A=1, \ldots, N$.

\section{Equations of the asymptotic models}

It can be observed that neglecting in equations (21), (22) or (23) terms involving parameter $l$ the governing equations of the simplified averaged models can be obtained.

Hence, from equations (21) we arrive at:

$$
\begin{aligned}
& B_{\alpha \beta \gamma \delta} \varphi_{\gamma, \delta \beta}-\frac{1}{60} d^{2} \mu C_{\alpha \beta} \ddot{w}_{, \beta}-D_{\alpha \beta}\left(w_{, \beta}+\varphi_{\beta}\right)-\vartheta \ddot{\varphi}_{\alpha} \\
& \quad-\frac{1}{10} d^{2} K C_{\alpha \beta} w_{, \beta}-\frac{1}{10} d^{2} m C_{\alpha \beta} \ddot{w}_{, \beta}+\left(\frac{1}{24}-\frac{1}{4}\right) d^{2} K_{t} \varphi_{\alpha} \\
& \quad+\left(\frac{1}{24}-\frac{1}{4}\right) d^{2} m \ddot{\varphi}_{\alpha}=-\frac{1}{10} d^{2}\left[\left(q_{3}^{+}+q_{3}^{-}\right)_{, \beta}\right. \\
& \left.\quad+\frac{1}{12} d\left(q_{\gamma}^{+}+q_{\gamma}^{-}\right)_{, \gamma \beta}\right] C_{\alpha \beta}+\left(\frac{1}{12}-\frac{1}{2}\right) d\left(q_{\alpha}^{+}-q_{\alpha}^{-}\right), \\
& D_{\alpha \beta}\left(w_{, \beta \alpha}+\varphi_{\beta, \alpha}\right)-(\mu+m) \ddot{w}-K w-\frac{1}{24} d^{2} K_{t} \varphi_{\beta, \alpha} \delta_{\alpha \beta} \\
& \quad-\frac{1}{24} d^{2} m \ddot{\varphi}_{\beta, \alpha} \delta_{\alpha \beta}=-\frac{1}{12} d\left(q_{\beta, \alpha}^{+}-q_{\beta, \alpha}^{-}\right) \delta_{\alpha \beta}-\left(q_{3}^{+}+q_{3}^{-}\right), \\
& \quad-B_{\alpha \beta \gamma \delta} H_{\delta \beta}^{a b} \Phi_{\gamma}^{a}+\frac{1}{10} d^{2} C_{\alpha \beta} \underline{\tilde{K}_{\beta}^{b} w}+\frac{1}{10} d^{2} C_{\alpha \beta} \underline{\tilde{m}_{\beta}^{b} \ddot{w}}=0, \\
& \quad-D_{\alpha \beta} G_{\alpha \beta}^{A B} V^{A}+\frac{1}{24} d^{2} \underline{K_{t \alpha}^{B} \varphi_{\beta} \delta_{\alpha \beta}}+\frac{1}{24} d^{2} \underline{m_{\alpha}^{B} \ddot{\varphi}_{\beta} \delta_{\alpha \beta}}=0,
\end{aligned}
$$

where the effect of period lengths is not taken into account. Coefficients in the above equations are defined by formulas (20). Because equations (24) do not describe the effect of period lengths, the averaged model describe by these equations can be called the asymptotic model.

Similarly, from equations (22) we obtain:

$$
\begin{aligned}
& B_{\alpha \beta \gamma \delta} \varphi_{\gamma, \delta \beta}-\frac{1}{60} d^{2} \mu C_{\alpha \beta} \ddot{w}_{, \beta}-D_{\alpha \beta}\left(w_{, \beta}+\varphi_{\beta}\right)-\vartheta \ddot{\varphi}_{\alpha} \\
& \quad-\frac{1}{10} d^{2} K C_{\alpha \beta} w_{, \beta}+\left(\frac{1}{24}-\frac{1}{4}\right) d^{2} K_{t} \varphi_{\alpha} \\
& \quad=-\frac{1}{10} d^{2}\left[\left(q_{3}^{+}+q_{3}^{-}\right)_{, \beta}+\frac{1}{12} d\left(q_{\gamma}^{+}+q_{\gamma}^{-}\right)_{, \gamma \beta}\right] C_{\alpha \beta} \\
& \quad+\left(\frac{1}{12}-\frac{1}{2}\right) d\left(q_{\alpha}^{+}-q_{\alpha}^{-}\right), \\
& D_{\alpha \beta}\left(w_{, \beta \alpha}+\varphi_{\beta, \alpha}\right)-\mu \ddot{w}-K w-\frac{1}{24} d^{2} K_{t} \varphi_{\beta, \alpha} \delta_{\alpha \beta} \\
& \quad=-\frac{1}{12} d\left(q_{\beta, \alpha}^{+}-q_{\beta, \alpha}^{-}\right) \delta_{\alpha \beta}-\left(q_{3}^{+}+q_{3}^{-}\right), \\
& \quad-B_{\alpha \beta \gamma \delta} H_{\delta \beta}^{a b} \Phi_{\gamma}^{a}+\frac{1}{10} d^{2} C_{\alpha \beta} \underline{\tilde{K}_{\beta}^{b} w}=0,-D_{\alpha \beta} G_{\alpha \beta}^{A B} V^{A} \\
& \quad+\frac{1}{24} d^{2} \underline{K_{t \alpha}^{B} \varphi_{\beta}} \delta_{\alpha \beta}=0,
\end{aligned}
$$

which describe the averaged model called the asymptotic model without the inertia of the foundation.

Finally, neglecting in equations (23) terms with parameter $l$ we have:

$$
\begin{aligned}
& B_{\alpha \beta \gamma \delta} \varphi_{\gamma, \delta \beta}-\frac{1}{60} d^{2} \mu C_{\alpha \beta} \ddot{w}_{, \beta}-D_{\alpha \beta}\left(w_{, \beta}+\varphi_{\beta}\right) \\
& \quad-\frac{1}{10} d^{2} K C_{\alpha \beta} w_{, \beta}+\left(\frac{1}{24}-\frac{1}{4}\right) d^{2} K_{t} \varphi_{\alpha} \\
& \quad=-\frac{1}{10} d^{2}\left[\left(q_{3}^{+}+q_{3}^{-}\right)_{, \beta}+\frac{1}{12} d\left(q_{\gamma}^{+}+q_{\gamma}^{-}\right)_{, \gamma \beta}\right] C_{\alpha \beta} \\
& \quad+\left(\frac{1}{12}-\frac{1}{2}\right) d\left(q_{\alpha}^{+}-q_{\alpha}^{-}\right), \\
& D_{\alpha \beta}\left(w_{, \beta \alpha}+\varphi_{\beta, \alpha}\right)-\mu \ddot{w}-K w-\frac{1}{24} d^{2} K_{t} \varphi_{\beta, \alpha} \delta_{\alpha \beta} \\
& \quad=-\frac{1}{12} d\left(q_{\beta, \alpha}^{+}-q_{\beta, \alpha}^{-}\right) \delta_{\alpha \beta}-\left(q_{3}^{+}+q_{3}^{-}\right), \\
& \quad-B_{\alpha \beta \gamma \delta} H_{\delta \beta}^{a b} \Phi_{\gamma}^{a}+\frac{1}{10} d^{2} C_{\alpha \beta} \tilde{K}_{\beta}^{b} w=0,-D_{\alpha \beta} G_{\alpha \beta}^{A B} V^{A} \\
& \quad+\frac{1}{24} d^{2} K_{t \alpha}^{B} \varphi_{\beta} \delta_{\alpha \beta}=0
\end{aligned}
$$

describing the averaged model called the asymptotic model without the inertia of the foundation and the rotational inertia of the plate.

\section{Summary}

Using the tolerance averaging technique, which was proposed for periodic structures by Woźniak and Wierzbicki [25] and summarized in the book edited by 
Woźniak et al. [24], the governing equations with constant coefficients of a non-asymptotic averaged model for medium thickness plates resting on a periodic Winkler's foundation are derived. This model, called the tolerance model, makes it possible to investigate the effect of period lengths on vibrations of these structures. Moreover, the derived model takes into account the effect of stress $S_{33}$, on the contrary to the model shown by Jędrysiak and Paś [15].

Summarizing, it can be observed that:

1. The proposed tolerance model is governed by equations with terms dependent explicitly on parameter $l$ (being the diameter of the periodicity cell). Hence, certain phenomena in dynamic problems, related to the internal periodic structure of the system of the plate and the foundation, can be investigated in the framework of this model.

2. Neglecting some terms in the governing equations, describing different effects related to the plate or the foundation, we obtain averaged models, which make it possible to analyse dynamical problems of systems under consideration on different levels of accuracy.

Some applications of the proposed models to dynamic problems of medium thickness plates resting on a periodic Winkler's foundation will be presented in the forthcoming papers.

Open Access This article is distributed under the terms of the Creative Commons Attribution License which permits any use, distribution, and reproduction in any medium, provided the original author(s) and the source are credited.

\section{References}

1. Ambartsumyan SA (1969) Theory of anisotropic plates. Technical Publishing Co., Stamford

2. Baron E (2003) On dynamic behaviour of medium-thickness plates with uniperiodic structure. Arch Appl Mech 73: 505-516

3. Bensoussan A, Lions J-L, Papanicolaou G (1978) Asymptotic analysis for periodic structures. North-Holland, Amsterdam

4. Bolle L (1947) Contribution au probleme lineaire deflexion d'une plaque elastique. Bull. Tech. Suisse Rom. 73(21): 281-285 (in French)

5. Caillerie D (1984) Thin elastic and periodic plates. Math. Methods Appl. Sci. 6:159-191

6. Cielecka I, Jędrysiak J (2006) A non-asymptotic model of dynamics of honeycomb lattice-type plates. J. Sound Vib 296:130-149
7. Dell'Isola F, Rosa L, Woźniak Cz (1998) A micro-structural continuum modelling compacting fluid-saturated grounds. Acta Mech 127:165-182

8. Domagalski Ł, Jędrysiak J (2012) On the elastostatics of thin periodic plates with large deflections. Meccanica 47(7):1659-1671. doi:10.1007/s11012-012-9546-1

9. Hencky H (1947) Über die Berücsichtigung der Schubverzerrung in ebenen Platten. Ing Arch 16:72-76 (in German)

10. Jemielita G (2001) Elastic plates theories, Chapter III. In: Woźniak C (ed) Mechanics of elastic plates and shells. PWN, Warsaw (in Polish)

11. Jędrysiak J (1999) Dynamics of thin periodic plates resting on a periodically inhomogeneous Winkler foundation. Arch Appl Mech 69:345-356

12. Jędrysiak J (2003) Free vibrations of thin periodic plates interacting with an elastic periodic foundation. Int $\mathbf{J}$ Mech Sci 45(8): 1411-1428

13. Jędrysiak J (2007) The tolerance averaging model of dynamic stability of thin plates with one-directional periodic structure. Thin-Walled Struct 45:855-860

14. Jędrysiak J (2009) Higher order vibrations of thin periodic plates. Thin-Walled Struct 47(8-9):890-901

15. Jędrysiak J, Paś A (2005) On the modelling of medium thickness plates interacting with a periodic Winkler's subsoil, Electr J Polish Agric Univ Civil Eng, 8, 4, (www.ejpau. media.pl)

16. Kaźmierczak M, Jędrysiak J (2011) Tolerance modelling of vibrations of thin functionally graded plates. Thin-Walled Struct 49:1295-1303. doi:10.1016/j.tws.2011.05.001

17. Kohn RV, Vogelius M (1984) A new model for thin plates with rapidly varying thickness. Int J Solids Struct 20: 333-350

18. Matysiak SJ, Nagórko W (1989) Microlocal parameters in the modelling of microperiodic plates. Ing Arch 59:434-444

19. Michalak B (2000) Vibrations of plates with initial geometrical periodical imperfections interacting with a periodic elastic foundation. Arch Appl Mech 70:508-518

20. Michalak B, Wirowski A (2012) Dynamic modelling of thin plate made of certain functionally graded materials. Meccanica 47(6):1487-1498

21. Tahouneh V, Naei MH (2014) A novel 2-D six-parameter power-law distribution for three-dimensional dynamic analysis of thick multi-directional functionally graded rectangular plates resting on a two-parameter elastic foundation. Meccanica 49(1):91-109

22. Tomczyk B (2007) A non-asymptotic model for the stability analysis of thin biperiodic cylindrical shells. Thin-Walled Struct 45:941-944

23. Vlasov WZ, Leontiev NN (1960) Beams, plates and shells on rigid subsoil. Gos. Izd. Fiz.-Mat. Lit., Moskva (in Russian)

24. Cz Woźniak, Michalak B, Jędrysiak J (eds) (2008) Thermomechanics of microheterogeneous solids and structures. Tolerance averaging approach. Wydawnictwo Politechniki Łódzkiej, Łódź

25. Cz Woźniak, Wierzbicki E (2000) Averaging techniques in thermomechanics of composite solids. Wydawnictwo Politechniki Częstochowskiej, Częstochowa

26. Kumar Yajuvindra, Lal R (2012) Vibrations of nonhomogeneous orthotropic rectangular plates with bilinear thickness variation resting on Winkler foundation. Meccanica 47(4):893-915 